\title{
Weyl-Dirac zero mode for calorons
}

\author{
Margarita García Pérez, ${ }^{1}$ Antonio González-Arroyo, ${ }^{1,2}$ Carlos Pena, ${ }^{1}$ and Pierre van Baal ${ }^{3}$ \\ ${ }^{1}$ Departamento de Física Teórica C-XI, Universidad Autónoma de Madrid, 28049 Madrid, Spain \\ ${ }^{2}$ Instituto de Física Teórica C-XVI, Universidad Autónoma de Madrid, 28049 Madrid, Spain \\ ${ }^{3}$ Instituut-Lorentz for Theoretical Physics, University of Leiden, P.O. Box 9506, NL-2300 RA Leiden, The Netherlands
}

(Received 4 May 1999; published 18 June 1999)

\begin{abstract}
We give the analytic result for the fermion zero mode of the $S U(2)$ calorons with a nontrivial holonomy. It is shown that the zero mode is supported on only one of the constituent monopoles. We discuss some of its implications. [S0556-2821(99)50513-4]
\end{abstract}

PACS number(s): 11.10.Wx, 14.80.Hv

\section{INTRODUCTION}

In this paper, we give the exact expression for the $S U(2)$ fermion zero mode in the field of the infinite volume caloron with a nontrivial holonomy and unit charge. Study of the gauge field configurations had, somewhat surprisingly, revealed that at nontrivial holonomy calorons have two Bogomol'nyi-Prasad-Sommerfield (BPS) monopoles $[N$ for $S U(N)]$ as their constituents [1,2,3]. For the HarringtonShepard [4] solution with trivial holonomy, this is hidden because one of the constituents is massless (it can be removed by a singular gauge transformation to show that the caloron for a large scale parameter becomes a single BPS monopole [5]). We find that, for calorons with wellseparated constituents, the fermion zero mode is entirely supported on one of them. In itself it is not surprising that the zero mode is correlated to the monopole constituents. Independently, this observation was recently also made for gluino zero modes in the context of supersymmetric gauge theories [6]. Gluinos are in the adjoint representation of the gauge group, such that there are four zero modes that can be split in pairs associated with each of the two constituents [6]. However, for the Dirac fermion, there is only one zero mode. To understand the "affinity" of the zero mode to only one of the two monopoles, we will analyze in some detail what distinguishes them.

Calorons are characterized by the (fixed) holonomy $[1,7]$. In the gauge in which $A_{\mu}(x)$ is periodic, this holonomy is given by

$$
\mathcal{P}_{\infty}=\lim _{|\vec{x}| \rightarrow \infty} \mathrm{P} \exp \left(\int_{0}^{\beta} A_{0}(t, \vec{x}) d t\right) \equiv \exp (2 \pi i \vec{\omega} \cdot \vec{\tau})
$$

Solutions are simplest in the so-called "algebraic' gauge, for which

$$
\begin{aligned}
& A_{\mu}(t+\beta, \vec{x})=\mathcal{P}_{\infty} A_{\mu}(t, \vec{x}) \mathcal{P}_{\infty}^{-1}, \\
& \Psi_{z}^{ \pm}(t+\beta, \vec{x})= \pm \mathcal{P}_{\infty} \Psi_{z}^{ \pm}(t, \vec{x}) .
\end{aligned}
$$

We will generalize the problem of finding the fermion zero mode in the field of the caloron with nontrivial holonomy, by adding a curvature free Abelian field, which forms the basis for the Nahm transformation [8]. Why this is useful will be evident from the construction. The equation to be solved is

$$
\bar{D}_{z} \Psi_{z}(x) \equiv \bar{\sigma}_{\mu}\left[\partial_{\mu}+A_{\mu}(x)-2 \pi i z_{\mu}\right] \Psi_{z}(x)=0,
$$

with $\bar{\sigma}_{\mu}=\sigma_{\mu}^{\dagger}=(1,-i \vec{\tau})$ and $\tau_{a}$ the Pauli matrices. For calorons, defined on $R^{3} \times S^{1}$, i.e., at finite temperature $1 / \beta$, one can choose $z_{1}=z_{2}=z_{3}=0$ [the plane-wave factor $\exp (2 \pi i \vec{z} \cdot \vec{x})$ does not affect the boundary conditions or the normalization of the zero mode and can be used to remove the $\vec{z}$ dependence]. But $z=z_{0}$ will be arbitrary (it has a dual period of $1 / \beta)$. The zero modes are represented as twocomponent spinors in the (chiral) Weyl decomposition for massless Dirac fermions.

\section{ADHM CONSTRUCTION}

The construction of the zero mode is best done in the Atiyah-Drinfeld-Hitchin-Manin (ADHM) formalism [9]. We will be brief in reviewing this formalism, further details can be found in Refs. $[1,10,11,12]$. In general the ADHM construction involves an operator $\Delta(x)$ [the "dual" of Eq. (3)], whose normalized zero mode, $\Delta^{\dagger}(x) v(x)=0$, gives the gauge field as $A_{\mu}(x)=v^{\dagger}(x) \partial_{\mu} v(x)$. For $S U(2)$ instantons of charge $k$, one has $\Delta^{\dagger}=\left(\lambda^{\dagger}, B^{\dagger}-x^{\dagger}\right)$, with $x^{\dagger}=x_{\mu} \bar{\sigma}_{\mu}, \lambda$ a $k$ dimensional row vector and $B$ a $k \times k$ symmetric matrix, all with values in the quaternions $\left(\lambda_{i I}^{l}, B_{I J}^{l, m}=B_{I J}^{m, l}\right.$, with $l, m$ $=1, \ldots, k$ "charge" and $I, J=1,2$ spinor indices, whereas $i=1,2$ is a color index). Introducing the row vector $u^{\dagger}(x)$ $\equiv \lambda(B-x)^{-1}$ and the scalar (real quaternion) $\phi(x)=1$ $+u^{\dagger}(x) u(x)$, it can be shown $[10,11,12]$ that the instanton gauge field and the $k$ zero modes are given by

$$
\begin{gathered}
A_{\mu}(x)=\phi^{-1}(x) \operatorname{Im}\left(u^{\dagger}(x) \partial_{\mu} u(x)\right), \\
\Psi_{i J}^{l}(x)=\pi^{-1} \phi^{-1 / 2}(x)\left(u^{\dagger}(x) f_{x}\right)_{i I}^{l} \varepsilon_{I J},
\end{gathered}
$$

where $f_{x}$ is the matrix inverse, or Green's function,

$$
f_{x}=\left((B-x)^{\dagger}(B-x)+\lambda^{\dagger} \lambda\right)^{-1} .
$$

Essential in the ADHM construction is that $\lambda$ and $B$ satisfy a quadratic constraint, which is equivalent to $f_{x}$ being a symmetric matrix whose imaginary quaternion components van- 
ish, $\left(f_{x}\right)_{I J}^{l, m} \equiv f_{x}^{l, m} \delta_{I J}$. From this alone, it can be proven that the gauge field is self-dual and that the $\Psi^{l}(x)$ are zero modes, $\bar{D} \Psi^{l}(x) \equiv \bar{\sigma}_{\mu}\left(\partial_{\mu}+A_{\mu}(x)\right) \Psi^{l}(x)=0$. Its proper normalization and the topological charge are read off from the remarkable results $[10,11,12]$

$$
\begin{gathered}
\Psi^{l}(x)^{\dagger} \Psi^{m}(x)=-(2 \pi)^{-2} \partial_{\mu}^{2} f_{x}^{l, m}, \\
\operatorname{Tr} F_{\mu \nu}^{2}(x)=-\partial_{\mu}^{2} \partial_{\nu}^{2} \log \operatorname{det} f_{x},
\end{gathered}
$$

using $\lim _{|x| \rightarrow \infty} f_{x}^{l, m}=\delta^{l, m}|x|^{-2}$. Before addressing the explicit form of these expressions for the caloron with nontrivial holonomy, we perform one further simplification (for the details follow Eqs. (21)-(29) in Ref. [1], see also Ref. [10]),

$$
\begin{gathered}
A_{\mu}(x)=\frac{1}{2} \phi(x) \partial_{\nu}\left(\lambda \bar{\eta}_{\mu \nu} f_{x} \lambda^{\dagger}\right), \\
\Psi_{i J}^{l}(x)=(2 \pi)^{-1} \phi^{1 / 2}(x) \partial_{\mu}\left(\lambda f_{x} \bar{\sigma}_{\mu}\right)_{i I}^{l} \varepsilon_{I J},
\end{gathered}
$$

where the anti-self-dual 't Hooft tensor $\bar{\eta}$ is defined by $\bar{\eta}_{0 j}^{i}$ $=-\bar{\eta}_{j 0}^{i}=\delta_{i j}$ and $\bar{\eta}_{j k}^{i}=\varepsilon_{i j k}$ (furthermore, $\varepsilon_{12}=1$ and with our conventions of $\left.t=x_{0}, \varepsilon_{0123}=-1\right)$.

The caloron with nontrivial holonomy is found by imposing boundary conditions to compactify time to a circle $u^{l+1}(t+\beta, \vec{x})=u^{l}(t, \vec{x}) \mathcal{P}_{\infty}^{\dagger}$, which is easily seen to give the correct boundary conditions for the gauge field. For the general form of $\lambda$ and $B$ which respect this symmetry, see Ref. [1]. Note that now the index $l$ runs over the set of all integers; the $R^{4}$ configuration with these boundary conditions has infinite topological charge (unit topological charge per time period). To obtain the zero mode with the appropriate boundary condition, we note that with Eq. (4)

$$
\hat{\Psi}_{z}(x) \equiv \sum_{l} e^{-2 \pi i l \beta z} \Psi^{l}(x)
$$

satisfies the boundary condition $\hat{\Psi}_{z}(t+\beta, \vec{x})$ $=e^{-2 \pi i \beta z} \mathcal{P}_{\infty} \hat{\Psi}_{z}(t, \vec{x})$ and satisfies $\bar{D} \hat{\Psi}_{z}(x)=0$ for all $z$. The general solution of the Weyl equation, Eq. (3), with both periodic and antiperiodic boundary conditions is now easily found (for simplicity, we put $\beta=1$ )

$$
\Psi_{z}^{+}(x)=e^{2 \pi i z t} \hat{\Psi}_{z}(x), \quad \Psi_{z}^{-}(x)=e^{2 \pi i z t} \hat{\Psi}_{z+1 / 2}(x) .
$$

In particular, $\Psi^{-}(x)=\Psi_{0}^{-}(x)=\hat{\Psi}_{1 / 2}(x)$ is the, for finite temperature, physically relevant chiral zero mode in the background of a caloron, whereas $\Psi^{+}(x)=\Psi_{0}^{+}(x)=\hat{\Psi}_{0}(x)$ is relevant for compactifications.

\section{NAHM-FOURIER TRANSFORMATION}

The interpretation of the "charge" index as a Fourier index, as suggested by the construction of the caloron zero mode, has been essential for solving the quadratic ADHM constraint in the presence of nontrivial holonomy. It maps the ADHM construction to the Nahm formalism, in which, furthermore, $f_{x}$ is solved in terms of a quantum mechanics problem on the circle $(z \in[0,1])$ with a piecewise constant potential and delta function singularities determined by the holonomy [1]. The relevant quantities involved are

$$
\hat{f}_{x}\left(z, z^{\prime}\right)=\sum_{l, m} f_{x}^{l, m} e^{2 \pi i\left(l z-m z^{\prime}\right)}, \hat{\lambda}(z)=\sum_{l} \lambda^{l} e^{-2 \pi i l z},
$$

where matrix multiplication is replaced by convolution in the usual sense. The solution of the ADHM constraint implies that $\hat{\lambda}(z)$ is the sum of two delta functions. Together with the explicit expression for the Green's function $\hat{f}_{x}\left(z, z^{\prime}\right)$ as given in Eqs. (47)-(49) of Ref. [1], the zero mode reads

$$
\hat{\Psi}_{z}(x)=(2 \pi)^{-1} \phi^{1 / 2}(x) \partial_{\mu}\left(\int_{0}^{1} d z^{\prime} \hat{\lambda}\left(z^{\prime}\right) \hat{f}_{x}\left(z^{\prime}, z\right) \bar{\sigma}_{\mu}\right)_{i I} \varepsilon_{I J}
$$

compare Eq. (4). Whereas Eq. (6) yields

$$
\hat{\Psi}_{z^{\prime}}^{\dagger}(x) \hat{\Psi}_{z}(x)=-(2 \pi)^{-2} \partial_{\mu}^{2} \hat{f}_{x}\left(z^{\prime}, z\right) .
$$

\section{EXPLICIT EXPRESSIONS} [1]

Using the classical scale invariance to put $\beta=1$, one has

$$
\begin{gathered}
s(x)=-\frac{1}{2} \operatorname{Tr} F_{\mu \nu}^{2}(x)=-\frac{1}{2} \partial_{\mu}^{2} \partial_{\nu}^{2} \log \psi(x), \\
\psi(x)=\hat{\psi}(\vec{x})-\cos (2 \pi t), \quad \hat{\psi}(\vec{x})=\frac{1}{2} \operatorname{tr}\left(\mathcal{A}_{2} \mathcal{A}_{1}\right),
\end{gathered}
$$

where

$$
\mathcal{A}_{m} \equiv \frac{1}{r_{m}}\left(\begin{array}{cc}
r_{m} & \left|\vec{y}_{m}-\vec{y}_{m+1}\right| \\
0 & r_{m+1}
\end{array}\right)\left(\begin{array}{cc}
c_{m} & s_{m} \\
s_{m} & c_{m}
\end{array}\right)
$$

Noting that $r_{3} \equiv r_{1}$ and $\vec{y}_{3} \equiv \vec{y}_{1}$, we defined $r_{m}=\left|\vec{x}-\vec{y}_{m}\right|$, with $\vec{y}_{m}$ the position of the $m$ th constituent monopole, which can be assigned a mass $8 \pi^{2} \nu_{m}$, where $\nu_{1}=2 \omega$ and $\nu_{2}$ $=2 \bar{\omega} \equiv 1-2 \omega . \quad$ Furthermore, $\quad c_{m} \equiv \cosh \left(2 \pi \nu_{m} r_{m}\right), \quad s_{m}$ $\equiv \sinh \left(2 \pi \nu_{m} r_{m}\right)$, and $\pi \rho^{2}=\left|\vec{y}_{2}-\vec{y}_{1}\right|$.

New is the result for the zero-mode density

$$
\begin{aligned}
& \left|\Psi^{-}(x)\right|^{2}=-(2 \pi)^{-2} \partial_{\mu}^{2} \hat{f}_{x}\left(\frac{1}{2}, \frac{1}{2}\right), \\
& \left|\Psi^{+}(x)\right|^{2}=-(2 \pi)^{-2} \partial_{\mu}^{2} \hat{f}_{x}(0,0),
\end{aligned}
$$

defined by [1]

$$
\begin{aligned}
\hat{f}_{x}\left(\frac{1}{2}, \frac{1}{2}\right)= & \frac{\pi}{r_{1} r_{2} \psi(x)}\left(s_{2}\left[r_{1} c_{1}+\pi \rho^{2} s_{1}\right]+r_{2} s_{1}\right. \\
& \left.+\frac{c_{2}-1}{r_{2}}\left[\pi \rho^{2} r_{1} c_{1}+\frac{1}{2}\left(r_{1}^{2}+r_{2}^{2}+\pi^{2} \rho^{4}\right) s_{1}\right]\right),
\end{aligned}
$$




$$
\begin{aligned}
\hat{f}_{x}(0,0)= & \frac{\pi}{r_{1} r_{2} \psi(x)}\left(s_{1}\left[r_{2} c_{2}+\pi \rho^{2} s_{2}\right]+r_{1} s_{2}\right. \\
& \left.+\frac{c_{1}-1}{r_{1}}\left[\pi \rho^{2} r_{2} c_{2}+\frac{1}{2}\left(r_{1}^{2}+r_{2}^{2}+\pi^{2} \rho^{4}\right) s_{2}\right]\right) .
\end{aligned}
$$

By a suitable combination of a constant gauge transformation, spatial rotation, and translation, we can arrange both $\vec{\omega}=(0,0, \omega)$ and the constituents at $\vec{y}_{1}=\left(0,0, \nu_{2} \pi \rho^{2}\right)$ and $\vec{y}_{2}$ $=\left(0,0,-\nu_{1} \pi \rho^{2}\right)$. For this choice, we find

$$
\begin{aligned}
A_{\mu}(x)= & \frac{i}{2} \bar{\eta}_{\mu \nu}^{3} \tau_{3} \partial_{\nu} \log \phi(x) \\
& +\frac{i}{2} \phi(x) \operatorname{Re}\left(\left(\bar{\eta}_{\mu \nu}^{1}-i \bar{\eta}_{\mu \nu}^{2}\right)\left(\tau_{1}+i \tau_{2}\right) \partial_{\nu} \chi(x)\right),
\end{aligned}
$$

$$
\begin{gathered}
\Psi_{1 I}^{-}(x)=(2 \pi)^{-1} \rho \phi^{1 / 2}(x)\left(\begin{array}{c}
\partial_{2}+i \partial_{1} \\
\partial_{0}-i \partial_{3}
\end{array}\right) \hat{f}_{x}\left(\omega, \frac{1}{2}\right), \\
\Psi_{2 I}^{-}(x)=\Psi_{1 J}^{-}(x)^{*} \varepsilon_{J I}, \\
\Psi_{1 I}^{+}(x)=(2 \pi)^{-1} \rho \phi^{1 / 2}(x)\left(\begin{array}{c}
\partial_{2}+i \partial_{1} \\
\partial_{0}-i \partial_{3}
\end{array}\right) \hat{f}_{x}(\omega, 0), \\
\Psi_{2 I}^{+}(x)=\Psi_{1 J}^{+}(x)^{*} \varepsilon_{J I},
\end{gathered}
$$

with $\quad \phi^{-1}(x)=1-\left[\pi \rho^{2} / \psi(x)\right]\left(s_{1} c_{2} / r_{1}+s_{2} c_{1} / r_{2}\right.$ $\left.+\pi \rho^{2} s_{1} s_{2} / r_{1} r_{2}\right)$ and $\chi(x)=\left[\pi \rho^{2} / \psi(x)\right]\left(e^{-2 \pi i t}\left(s_{1} / r_{1}\right)\right.$ $\left.+s_{2} / r_{2}\right) e^{2 \pi i \nu_{1} t}$, and

$$
\begin{aligned}
\hat{f}_{x}\left(\omega, \frac{1}{2}\right)= & \frac{\pi e^{\pi i \nu_{1} t}}{r_{1} r_{2} \psi(x)}\left\{\left(e^{\pi i t} r_{1}\right.\right. \\
& \left.+e^{-\pi i t}\left[\pi \rho^{2} s_{1}+r_{1} c_{1}\right]\right) \sinh \left(\pi r_{2} \nu_{2}\right) \\
& \left.+e^{-\pi i t} r_{2} s_{1} \cosh \left(\pi r_{2} \nu_{2}\right)\right\}, \\
\hat{f}_{x}(\omega, 0)= & \frac{\pi e^{-\pi i \nu_{2} t}}{r_{1} r_{2} \psi(x)}\left\{\left(e^{-\pi i t} r_{2}\right.\right. \\
& \left.+e^{\pi i t}\left[\pi \rho^{2} s_{2}+r_{2} c_{2}\right]\right) \sinh \left(\pi r_{1} \nu_{1}\right) \\
& \left.+e^{\pi i t} r_{1} s_{2} \cosh \left(\pi r_{1} \nu_{1}\right)\right\} .
\end{aligned}
$$

\section{PROPERTIES OF THE ZERO MODE}

The gauge field has a symmetry under the antiperiodic gauge transformation $g(x)=\exp (\pi i t \hat{\omega} \cdot \vec{\tau})$, which changes the sign of the holonomy, $\mathcal{P}_{\infty} \rightarrow-\mathcal{P}_{\infty}$, or $\omega \leftrightarrow \bar{\omega}=\frac{1}{2}-\omega$. An antiperiodic gauge transformation does, however, not leave fermions invariant, and indeed it interchanges $\Psi_{z}^{+}(x)$ and $\Psi_{z}^{-}(x)$. To preserve the special choice of parametrization presented above, the change of sign in the holonomy, which interchanges $\nu_{1}$ and $\nu_{2}$, is also accompanied by an interchange of the constituent locations. This indeed leaves the action density invariant. That the zero mode clearly distin-

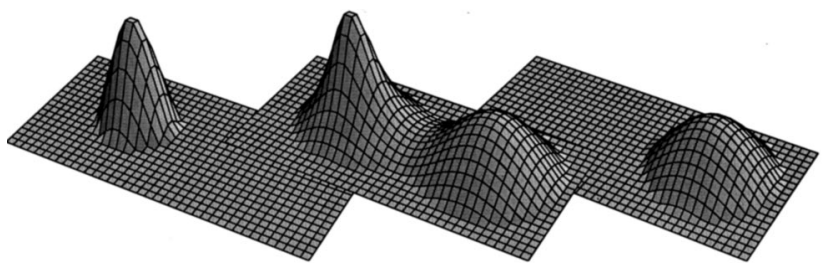

FIG. 1. For the two figures on the sides, we plot on the same scale the logarithm of the zero mode densities (cutoff below $1 / e^{5}$ ) for $\omega=1 / 8$ (left $\Psi^{-}$/right $\Psi^{+}$) and $\omega=3 / 8$ (right $\Psi^{-} /$left $\Psi^{+}$), with $\beta=1$ and $\rho=1.2$. In the middle figure, we show for the same parameters (both choices of $\omega$ give the same action density), the logarithm of the action density (cutoff below $1 / 2 e^{2}$ ).

guishes between the two cases becomes evident in the static limit, $\rho \rightarrow \infty$ (or equivalently $\beta \rightarrow 0$ ), in which case the zero mode is completely localized on one of the constituent monopoles, as follows from [compare Eq. (16)]

$$
\begin{aligned}
& \lim _{\rho \rightarrow \infty}\left|\Psi^{-}(x)\right|^{2}=-\partial_{\mu}^{2}\left(\frac{\tanh \left(\pi r_{2} \nu_{2}\right)}{4 \pi r_{2}}\right), \\
& \lim _{\rho \rightarrow \infty}\left|\Psi^{+}(x)\right|^{2}=-\partial_{\mu}^{2}\left(\frac{\tanh \left(\pi r_{1} \nu_{1}\right)}{4 \pi r_{1}}\right) .
\end{aligned}
$$

Under the antiperiodic gauge transformation, the antiperiodic zero mode becomes periodic. The new antiperiodic zero mode is now completely localized on the other constituent monopole [this is consistent with the fact that $\hat{f}_{x}(0,0)$ can be obtained from $\hat{f}_{x}\left(\frac{1}{2}, \frac{1}{2}\right)$ by interchanging $r_{1}$ and $\nu_{1}$ with $r_{2}$ and $\nu_{2}$. Figure 1 illustrates these issues [13].

In the gauge where $A_{\mu}(x)$ is periodic for large $\rho$, one of the constituent monopole fields is completely time independent, whereas the other one has a time dependence that would result from a full rotation along the axis connecting the two constituents [1]. This is read off from

$$
\begin{aligned}
A_{\mu}^{\mathrm{per}}= & \frac{i}{2} \bar{\eta}_{\mu \nu}^{3} \tau_{3} \partial_{\nu} \log \phi \\
& +\frac{i}{2} \phi \operatorname{Re}\left[\left(\bar{\eta}_{\mu \nu}^{1}-i \bar{\eta}_{\mu \nu}^{2}\right)\left(\tau_{1}+i \tau_{2}\right)\left(\partial_{\nu}+4 \pi i \omega \delta_{\nu, 0}\right) \tilde{\chi}\right] \\
& +\delta_{\mu, 0} 2 \pi i \omega \tau_{3}, \\
\widetilde{\chi}= & e^{-4 \pi i t \omega} \chi \\
= & \frac{4 \pi \rho^{2}}{\left(r_{2}+r_{1}+\pi \rho^{2}\right)^{2}}\left(r_{2} e^{-2 \pi r_{2} \nu_{2}} e^{-2 \pi i t}+r_{1} e^{-2 \pi r_{1} \nu_{1}}\right) \\
& \times\left[1+\mathcal{O}\left(e^{-4 \pi \min \left(r_{1} \nu_{1}, r_{2} \nu_{2}\right)}\right)\right] .
\end{aligned}
$$

[Note that for large $\rho, \phi(x)$ becomes time independent.] This full rotation-which we will call the Taubes-winding-is responsible for the topological charge of the otherwise time independent monopole pair [14]. Under the antiperiodic gauge transformation that changes the sign of the holonomy, the Taubes-winding is supported by the other constituent. It has not gone unnoticed that the antiperiodic fermion zero 
mode is precisely localized on the monopole constituent that carries the Taubes-winding. Another way to distinguish the two constituent monopoles is by inspecting the Polyakov loop values at their centers. One finds -1 for the monopole line with the Taubes-winding and +1 for the other monopole line (this is correlated to the vanishing of the would-be Higgs field), see the Appendix of Ref. [15]. For trivial holonomy, $\omega=0$, the Polyakov loop is indeed -1 at the center of the Harrington-Shepard [4] caloron. Its zero mode, constructed before in Refs. [7,16], agrees with the results found here.

The association of the zero mode with the constituent that carries the Taubes-winding lends considerable support for the role of the monopole loops with Taubes-winding in QCD for chiral dynamics [1]. The precise embedding of these straight finite temperature monopole loops as curved monopole loops in flat space remains a nontrivial and challenging problem. Although it may seem contradictory to expect the zero mode with antiperiodic boundary conditions to be the relevant one, one should not forget that for a curved monopole loop, the spin frame makes also one full rotation due to the bending of the loop, thereby most likely providing the compensating sign flip.

\section{ACKNOWLEDGMENTS}

We are grateful to Maxim Chernodub, Arjan Keurentjes, Valya Khoze, and Tamas Kovács for useful discussions and correspondence. A. Gonzalez-Arroyo and C. Pena acknowledge financial support by CICYT under Grant No. AEN971678. M. García Pérez acknowledges financial support by CICYT.
[1] T. C. Kraan and P. van Baal, Nucl. Phys. B533, 627 (1998).

[2] T. C. Kraan and P. van Baal, Phys. Lett. B 428, 268 (1998); 435, 389 (1998).

[3] K. Lee and P. Yi, Phys. Rev. D 56, 3711 (1997); K. Lee, Phys. Lett. B 426, 323 (1998); K. Lee and C. Lu, Phys. Rev. D 58, 025011 (1998).

[4] B. J. Harrington and H. K. Shepard, Phys. Rev. D 17, 2122 (1978); 18, 2990 (1978).

[5] P. Rossi, Nucl. Phys. B149, 170 (1979).

[6] T. J. Hollowood, V. V. Khoze, W. Lee, and M. P. Mattis, "Breakdown of cluster decomposition in instanton calculations of the gluino condensate," hep-th/9904116; N. M. Davies, T. J. Hollowood, V. V. Khoze, and M. P. Mattis, "Gluino condensate from magnetic monopoles in SUSY gluodynamics," hep-th/9905015.

[7] D. J. Gross, R. D. Pisarski, and L. G. Yaffe, Rev. Mod. Phys. 53, 43 (1983).

[8] W. Nahm, Phys. Lett. 90B, 413 (1980); Self-dual monopoles and calorons, Lecture Notes in Physics Vol. 201, edited by G.
Denardo (Springer, Berlin, 1984), p. 189.

[9] M. F. Atiyah, N. J. Hitchin, V. G. Drinfeld, and Yu. I. Manin, Phys. Lett. 65A, 185 (1978).

[10] E. F. Corrigan, D. B. Fairlie, S. Templeton, and P. Goddard, Nucl. Phys. B140, 31 (1978).

[11] H. Osborn, Nucl. Phys. B140, 45 (1978); Ann. Phys. (N.Y.) 135, 373 (1981).

[12] E. Corrigan and P. Goddard, Ann. Phys. (N.Y.) 154, 253 (1984).

[13] C-programmes for action/zero-mode densities and Polyakov loops can be found at http://www-lorentz.leidenuniv.nl/ vanbaal/Caloron.html.

[14] C. Taubes, in Progress in Gauge Field Theory, edited by G. 't Hooft et al. (Plenum, New York, 1984), p. 563.

[15] M. García Pérez, A. González-Arroyo, A. Montero, and P. van Baal, "Calorons on the lattice-a new perspective," hep-lat/9903022.

[16] N. Bilić, Phys. Lett. 97B, 107 (1980); A. González-Arroyo and Yu. A. Simonov, Nucl. Phys. B460, 429 (1996). 